\title{
Diversity Analysis of Bit-Interleaved Coded Multiple Beamforming
}

\author{
Hong Ju Park and Ender Ayanoglu \\ Center for Pervasive Communications and Computing \\ Department of Electrical Engineering and Computer Science \\ University of California, Irvine \\ Email: hjpark@uci.edu, ayanoglu@uci.edu
}

\begin{abstract}
In this paper, diversity analysis of bit-interleaved coded multiple beamforming (BICMB) is extended to the case of general spatial interleavers, removing a condition on their previously known design criteria and quantifying the resulting diversity order. The diversity order is determined by a parameter $Q_{\max }$ which is inherited from the convolutional code and the spatial de-multiplexer used in BICMB. We introduce a method to find this parameter by employing a transfer function approach as in finding the weight spectrum of a convolutional code. By using this method, several $Q_{\max }$ 's are shown and verified to be identical with the results from a computer searching program tracing paths on the trellis. The diversity analysis and the method to find the parameter are supported by simulation results.
\end{abstract}

\section{INTRODUCTION}

When the channel information is perfectly available at the transmitter, beamforming is an attractive technique to enhance the performance of a multi-input multi-output (MIMO) system [1]. The beamforming vectors are obtained by singular value decomposition (SVD) which is optimal in terms of minimizing the average bit error rate (BER) [2]. Single beamforming, which carries only one symbol at a time, was shown to achieve full diversity order of $N M$ where $N$ is the number of transmit antennas and $M$ is the number of receive antennas [3]. However, multiple beamforming, which increases the throughput by sending multiple symbols at a time, loses the full diversity order over flat fading channels.

To achieve the full diversity order as well as the full spatial multiplexing order, bit-interleaved coded multiple beamforming, combining bit-interleaved coded modulation (BICM) and multiple beamforming, was introduced in [4]. Design criteria for interleaving the coded sequence were provided such that each subchannel created by SVD is utilized at least once with a corresponding channel bit equal to 1 in an error event on the trellis diagram [4], [5]. BICMB with 1/2-rate convolutional encoder, a simple interleaver and soft-input Viterbi decoder was shown to have full diversity order when it is used in a $2 \times 2$ system with 2 streams. In this paper, the diversity order is analyzed even when the interleaver does not meet the design criteria. To determine the diversity order, the error events that dominate BER performance need to be found. We introduce a method to find the dominant error events by extending a method from convolutioinal code analysis to determine system performance, e.g., [6], into the analysis of the combination of the interleaver and the code.

The rest of this paper is organized as follows. A brief review of the BICMB system is given in Section $\amalg$ Section III introduces a method to find $\alpha$-vectors for a given convolutional code and the number of subchannel. Pair-wise error probability (PEP) analysis is given in Section IV Simulation results supporting the analysis are shown in Section $\mathrm{V}$. Finally, we end the paper with a conclusion in $\mathrm{VI}$.

\section{BICMB OVERVIEW}

The convolutional encoder, possibly combined with a perforation matrix for a high rate punctured code, generates the codeword $\mathbf{c}$ from the input packet. Then, the spatial demultiplexer distributes the coded bits into $S$ sequences, each of which is interleaved by an independent bit-wise interleaver. The interleaved sequences are mapped by Gray encoding onto the symbol sequences $\mathbf{y}$. A symbol belongs to a signal set $\chi \subset \mathbb{C}$ of size $|\chi|=2^{m}$, such as $2^{m}$-QAM, where $m$ is the number of input bits to the Gray encoder.

The MIMO channel $\mathbf{H} \in \mathbb{C}^{M \times N}$ is assumed to be quasistatic, Rayleigh, and flat fading, and perfectly known to both the transmitter and the receiver. The beamforming vectors are determined by the singular value decomposition of the MIMO channel, i.e., $\mathbf{H}=\mathbf{U} \boldsymbol{\Lambda} \mathbf{V}^{\mathbf{H}}$ where $\mathbf{U}$ and $\mathbf{V}$ are unitary matrices, and $\boldsymbol{\Lambda}$ is a diagonal matrix whose $s^{t h}$ diagonal element, $\lambda_{s} \in \mathbb{R}$, is a singular value of $\mathbf{H}$ with decreasing order. When $S$ symbols are transmitted at the same time, then the first $S$ vectors of $\mathbf{U}$ and $\mathbf{V}$ are chosen to be used as beamforming matrices at the receiver and the transmitter, respectively. Let's denote the first $S$ vectors of $\mathbf{U}$ and $\mathbf{V}$ as $\tilde{\mathbf{U}}$ and $\tilde{\mathbf{V}}$. The system input-output relation at the $k^{t h}$ time instant for a packet duration is written as

$$
\mathbf{r}_{k}=\tilde{\mathbf{U}}^{H} \mathbf{H} \tilde{\mathbf{V}} \mathbf{y}_{k}+\tilde{\mathbf{U}}^{H} \mathbf{n}_{k}
$$

where $\mathbf{y}_{k}$ is an $S \times 1$ vector of transmitted symbols, $\mathbf{r}_{k}$ is an $S \times 1$ vector of the detected symbols, and $\mathbf{n}_{k}$ is an additive white Gaussian noise vector with zero mean and variance $N_{0}=N / S N R$. On each $s^{t h}$ subchannel, finally, we get

$$
r_{k, s}=\lambda_{s} y_{k, s}+\tilde{n}_{k, s}
$$


where $r_{k, s}, y_{k, s}$, and $\tilde{n}_{k, s}$ are a detected symbol, a transmitted symbol, and a noise term, respectively. $\mathbf{H}$ is complex Gaussian with zero mean and unit variance, and to make the received signal-to-noise ratio $S N R$, the total transmitted power is scaled as $N$.

The location of $l^{t h}$ coded bit $c_{l}$ within the detected symbols is stored in a table $l \rightarrow(k, s, i)$, where $k, s$, and $i$ are time instant, subchannel, and bit position on a symbol, respectively. Let $\chi_{b}^{i} \subset \chi$ where $b \in\{0,1\}$ in the $i^{t h}$ bit position. By using the information in the table and the input-output relation in (2), the receiver calculates the ML bit metrics as

$$
\gamma^{i}\left(r_{k, s}, c_{l}\right)=\min _{y \in \chi_{c_{l}}^{i}}\left|r_{k, s}-\lambda_{s} y\right|^{2} .
$$

The combination of the ML bit metrics of (3) and $\tilde{\mathbf{U}}$ detector at the receiver is not the unique solution to get the optimum BER performance. Appropriate bit metrics corresponding to a linear detector, such as zero-forcing (ZF) or minimum mean square error (MMSE) detector, were shown to be equivalent to the bit metrics of (3) with $\tilde{\mathbf{U}}$ detector [7]. Finally, the ML decoder can make decisions according to the rule

$$
\hat{\mathbf{c}}=\arg \min _{\tilde{\mathbf{c}}} \sum_{l} \gamma^{i}\left(r_{k, s}, \tilde{c}_{l}\right) .
$$

\section{III. $\alpha$-SPECTRA}

The BER of a BICMB system is upper bounded by all the summations of each pairwise error probability for all the error events on the trellis [4], [5]. Therefore, the calculation of PEP for each error event is needed to analyze the diversity order of a given BICMB system. If the interleaver is properly designed such that the consecutive long coded bits are mapped onto distinct symbols, the PEP between the two codewords $\mathbf{c}$ and $\hat{\mathbf{c}}$ with Hamming distance $d_{H}$ is upper bounded as [4]

$$
\begin{aligned}
P(\mathbf{c} \rightarrow \hat{\mathbf{c}}) & =E[P(\mathbf{c} \rightarrow \hat{\mathbf{c}} \mid \mathbf{H})] \\
& \leq E\left[\frac{1}{2} \exp \left(-\frac{d_{\min }^{2} \sum_{s=1}^{S} \alpha_{s} \lambda_{s}^{2}}{4 N_{0}}\right)\right]
\end{aligned}
$$

where $d_{\min }$ is the minimum Euclidean distance in the constellation, and $\alpha_{s}$ denotes the number of usages of the $s^{\text {th }}$ subchannel of corresponding $d_{H}$ bits under consideration satisfying $\sum_{s=1}^{S} \alpha_{s}=d_{H}$. Since PEP is affected by the summation of the products between $\alpha_{s}$ and singular values as can be seen in (5), it is important to calculate the $\alpha$-vectors for each error path to have an insight into the diversity order behavior of a particular BICMB implementation.

We developed a method to calculate the $\alpha$-vectors for a convolutional code and interleaver combination. We will now illustrate this method with a simple example. Assume the system is composed of a 4 -state $1 / 2$-rate convolutional encoder and a spatial de-multiplexer rotating with an order of $a, b, c$, and $d$ which represent the streams. Fig. 11 represents a trellis diagram of this convolutional encoder for one period at the steady state. Since a convolutional code is linear, the all-

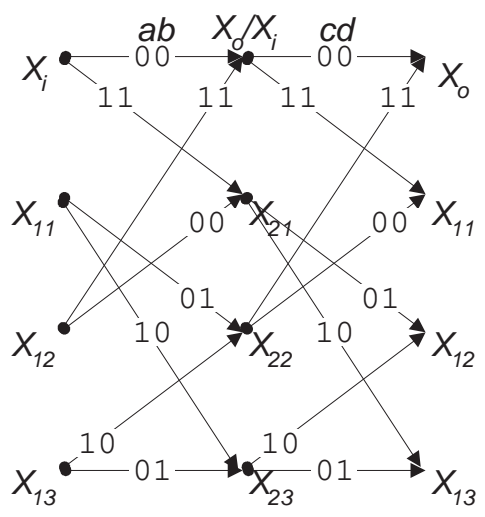

Fig. 1. Trellis of 4 -state $1 / 2$-rate convolutional code with 4 streams

zero codeword is assumed to be the input to the encoder. To find a transfer function of a convolutional code and a spatial de-multiplexer, we label the branches as a combination of $a^{\phi_{a}}$, $b^{\phi_{b}}, c^{\phi_{c}}$, and $d^{\phi_{d}}$, where the exponent $\phi_{i}$ denotes the number of usage of the subchannel $i$ which contributes to detecting the wrong branch by the detector. Additionally, $Z^{\phi_{z}}$, whose exponent satisfies $\phi_{Z}=\phi_{a}+\phi_{b}+\phi_{c}+\phi_{d}$, is included to get the relationship between the Hamming distance and $\alpha$ vector of an error event. Furthermore, the non-zero states are arbitrarily labeled $X_{11}$ through $X_{23}$, while the zero state is labeled as $X_{i}$ if branches split and $X_{o}$ if branches merge as shown in Fig. 1

Let's denote $\mathbf{x}=\left[\begin{array}{llllll}X_{11} & X_{12} & X_{13} & X_{21} & X_{22} & X_{23}\end{array}\right]^{T}$. Then, the state equations are given by the matrix equation

$$
\begin{aligned}
\mathbf{x} & =\mathbf{F x}+\mathbf{t} X_{i} \\
& =\left[\begin{array}{cccccc}
0 & 0 & 0 & 0 & 1 & 0 \\
0 & 0 & 0 & b Z & 0 & a Z \\
0 & 0 & 0 & a Z & 0 & b Z \\
0 & 1 & 0 & 0 & 0 & 0 \\
d Z & 0 & c Z & 0 & 0 & 0 \\
c Z & 0 & d Z & 0 & 0 & 0
\end{array}\right] \mathbf{x}+\left[\begin{array}{c}
c d Z^{2} \\
0 \\
0 \\
a b Z^{2} \\
0 \\
0
\end{array}\right] X_{i} .
\end{aligned}
$$

We also get

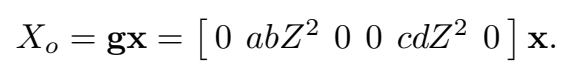

The transfer function is represented in closed form by using the method in [6] as

$$
\begin{aligned}
& \mathbf{T}(a, b, c, d, Z)=\mathbf{g}[\mathbf{I}-\mathbf{F}]^{-1} \mathbf{t}=\mathbf{g t}+\sum_{k=1}^{\infty} \mathbf{g} \mathbf{F}^{k} \mathbf{t} \\
& =Z^{5}\left(a^{2} b^{2} d+b c^{2} d^{2}\right) \\
& +Z^{6}\left(2 a^{2} b c^{2} d+a^{2} b^{2} d^{2}+b^{2} c^{2} d^{2}\right) \\
& +Z^{7}\left(a^{2} b^{3} c^{2}+2 a^{2} b^{2} c^{2} d+2 a^{2} b c^{2} d^{2}+\right. \\
& \left.\quad b^{3} c^{2} d^{2}+a^{2} b^{2} d^{3}+a^{2} c^{2} d^{3}\right) \\
& +Z^{8}\left(a^{4} b^{2} c^{2}+4 a^{2} b^{3} c^{2} d+4 a^{2} b^{2} c^{2} d^{2}+\right. \\
& \left.\quad b^{4} c^{2} d^{2}+a^{2} c^{4} d^{2}+4 a^{2} b c^{2} d^{3}+a^{2} b^{2} d^{4}\right)+\cdots
\end{aligned}
$$

where $[\mathbf{I}-\mathbf{F}]^{-1}$ can be expanded as $\mathbf{I}+\mathbf{F}+\mathbf{F}^{2}+\cdots$ through 
an infinite series of power of matrices. The weight spectrum, used for error performance analysis of convolutional codes, can be easily determined by $\left.\mathbf{T}(a, b, c, d, Z)\right|_{a=b=c=d=1}$ and can be compared with the literature [8], [9].

Assume that $a, b, c, d$ are assigned to be the stream numbers $1,2,3$, and 4 , respectively. We can then figure out from the transfer function that the $\alpha$-vectors of two error events with Hamming distance equal to 5 are [ [ $\left.\begin{array}{llll}2 & 2 & 0 & 1\end{array}\right]$ and $\left[\begin{array}{llll}0 & 1 & 2 & 2\end{array}\right]$. Besides, the vectors with $\alpha_{1}$ equal to 0 are easily found by choosing the terms composed of only $b, c$, and $d$, which are [0 1222 , $\left[\begin{array}{llll}0 & 2 & 2 & 2\end{array}\right],\left[\begin{array}{llll}0 & 3 & 2 & 2\end{array}\right]$, and [ $\left[\begin{array}{llll}0 & 4 & 2 & 2\end{array}\right]$. No vector is found which has $\alpha_{1}=\alpha_{2}=0$ or $\alpha_{1}=\alpha_{2}=\alpha_{3}=0$.

This method can be applied to any $K$-state $k_{c} / n_{c}$-rate convolutional code and $S$-stream BICMB system. If the spatial de-multiplexer is not a random switch for the whole packet, the period of the spatial de-multiplexer can be an integer multiple of least common multiples (LCM) of $n_{c}$ and $S$. Let's denote $P=\operatorname{LCM}\left(n_{c}, S\right)$ which means the number of coded bits for a minimum period. Then, the dimension of the vector $\mathbf{x}$ is $n P(K-1) k_{c} / n_{c}$ where $n$ is the integer multiple for a period of interest.

By using this method, transfer functions of a 4 -state $1 / 2$ rate $(5,7)$ convolutional code combined with several different de-multiplexers are shown in (10), (11), and (12). The spatial de-multiplexer used in $\mathbf{T}_{1}$ and $\mathbf{T}_{2}$ is a simple rotating switch on 2 and 3 subchannels, respectively. For $\mathbf{T}_{3}, i^{t h}$ coded bit is de-multiplexed into subchannel $s_{\bmod (i, 18)+1}$ where $s_{1}=\cdots$ $=s_{6}=1, s_{7}=\cdots=s_{12}=2, s_{13}=\cdots=s_{18}=3$ and $\bmod$ is a modular operation. Throughout the transfer functions, the variables $a, b$, and $c$ represent $1^{\text {st }}, 2^{\text {nd }}$, and $3^{\text {rd }}$ subchannel, respectively, in a decreasing order of singular values from the channel matrix.

$$
\begin{aligned}
& \mathbf{T}_{1}=Z^{5}\left(a^{2} b^{3}\right)+Z^{6}\left(a^{4} b^{2}+a^{2} b^{4}\right) \\
&+Z^{7}\left(3 a^{4} b^{3}+a^{2} b^{5}\right) \\
&+Z^{8}\left(a^{6} b^{2}+6 a^{4} b^{4}+a^{2} b^{6}\right) \\
&+Z^{9}\left(5 a^{6} b^{3}+10 a^{4} b^{5}+a^{2} b^{7}\right) \\
&+Z^{10}\left(a^{8} b^{2}+15 a^{6} b^{4}+15 a^{4} b^{6}+a^{2} b^{8}\right)+\cdots \\
& \mathbf{T}_{2}= Z^{5}\left(a^{2} b^{2} c+a^{2} b c^{2}+a b^{2} c^{2}\right) \\
&+ Z^{6}\left(a^{3} b^{2} c+a^{2} b^{3} c+a^{3} b c^{2}+\right. \\
&\left.a b^{3} c^{2}+a^{2} b c^{3}+a b^{2} c^{3}\right) \\
&+Z^{7}\left(2 a^{3} b^{3} c+2 a^{3} b^{2} c^{2}+2 a^{2} b^{3} c^{2}+\right. \\
&\left.2 a^{3} b c^{3}+2 a^{2} b^{2} c^{3}+2 a b^{3} c^{3}\right) \\
&+ Z^{8}\left(a^{5} b^{3}+a^{4} b^{3} c+a^{3} b^{4} c+2 a^{4} b^{2} c^{2}+\right. \\
& 3 a^{3} b^{3} c^{2}+2 a^{2} b^{4} c^{2}+a^{4} b c^{3}+3 a^{3} b^{2} c^{3}+ \\
& 3 a^{2} b^{3} c^{3}+a b^{4} c^{3}+b^{5} c^{3}+a^{3} b c^{4}+ \\
&\left.2 a^{2} b^{2} c^{4}+a b^{3} c^{4}+a^{3} c^{5}\right)+\cdots \\
& \\
& \mathbf{T}_{3}= Z^{5}\left(a^{5}+a^{3} b^{2}+a^{2} b^{3}+\right. \\
&\left.b^{5}+a^{3} c^{2}+b^{3} c^{2}+a^{2} c^{3}+b^{2} c^{3}+c^{5}\right) \\
&+Z^{6}\left(a^{4} b^{2}+3 a^{3} b^{3}+a^{2} b^{4}+a^{4} c^{2}+3 a^{2} b^{2} c^{2}+\right.
\end{aligned}
$$

where $\beta$ is a constant. Therefore, the PEP in (13) is upper bounded as

$$
P(\mathbf{c} \rightarrow \hat{\mathbf{c}}) \leq \gamma\left(\frac{d_{m i n}^{2} \alpha_{\tilde{Q}}}{4 N} S N R\right)^{-(M-\tilde{Q}+1)(N-\tilde{Q}+1)}
$$

where $\gamma$ is a constant.

Secondly, for the PEP calculation of the codewords resulting in more than one non-zero elements in an $\alpha$-vector, we need to calculate the marginal pdf for the eigenvalues corresponding to non-zero $\alpha$ 's. The closed form of the marginal pdf for 


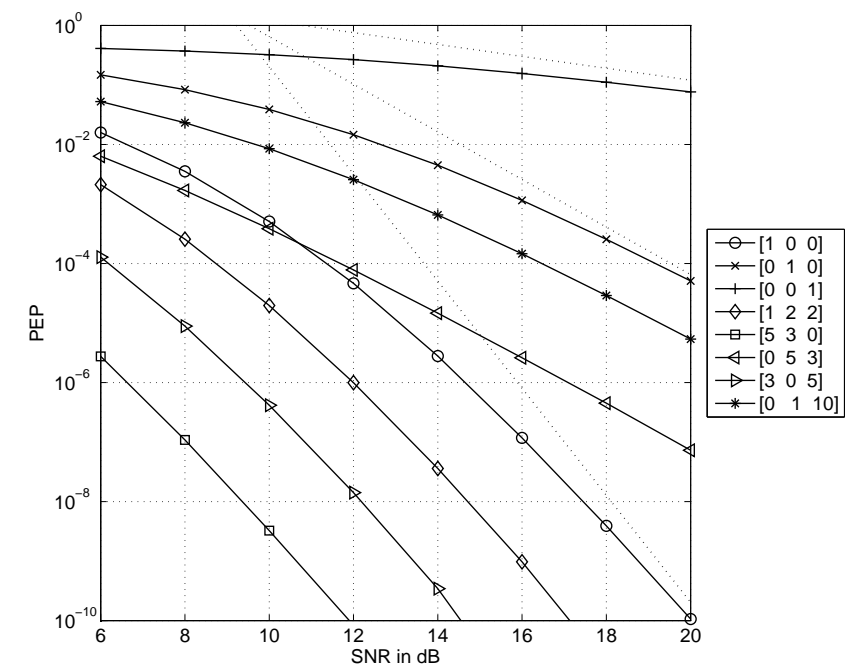

Fig. 2. Monte-Carlo Simulation Results for $3 \times 3 S=3$ case with 4-QAM

the concerned variables is not available analytically. However, one can calculate (5) via a computer and get an insight into the diversity associated with an error path. Fig. 2 shows the calculations of (5) corresponding to several specific $\alpha$ vectors through Monte-Carlo simulation. Based on these observations, we conclude that the exponent of PEP at high SNR is $(M-\hat{Q}+1)(N-\hat{Q}+1)$, where $\hat{Q}$ is an index that indicates the first non-zero element in the $\alpha$-vector. By taking both (15) and the observations in Fig. 2 into consideration, we express the exponent of PEP between two codewords as $(M-Q+1)(N-Q+1)$ where $Q$ is an index that indicates the first non-zero element in the $\alpha$-vector.

For a rate $k_{c} / n_{c}$ binary convolutional code and a fixed Grayencoded constellation labeling map in a BICMB system, BER $P_{b}$ can be bounded as

$$
P_{b} \leq \frac{1}{k_{c}} \sum_{d=d_{\text {free }}}^{\infty} \sum_{i=1}^{W_{I}(d)} g(d, Q(d, i), \chi)
$$

where $g(\cdot)$ is PEP corresponding to each error event, $W_{I}(d)$ denotes the total input weight of error events at Hamming distance $d$, and $Q$ is different for each error event. Since BER is dominated by PEP with the worst exponent term, the diversity order of a given BICMB system can be represented by

$$
O_{\text {diversity }}=\left(M-Q_{\max }+1\right)\left(N-Q_{\max }+1\right)
$$

where $Q_{\max }$ is the maximum $Q$ among the whole set of $Q$ 's corresponding to all of the error events.

\section{Simulation Results}

To show the validity of the diversity order analysis in Section IV by using the method in Section $\amalg$ to get $Q_{\max }$, BER against SNR are derived through a Monte-Carlo simulation. Throughout the simulation, 4-QAM modulation with Gray

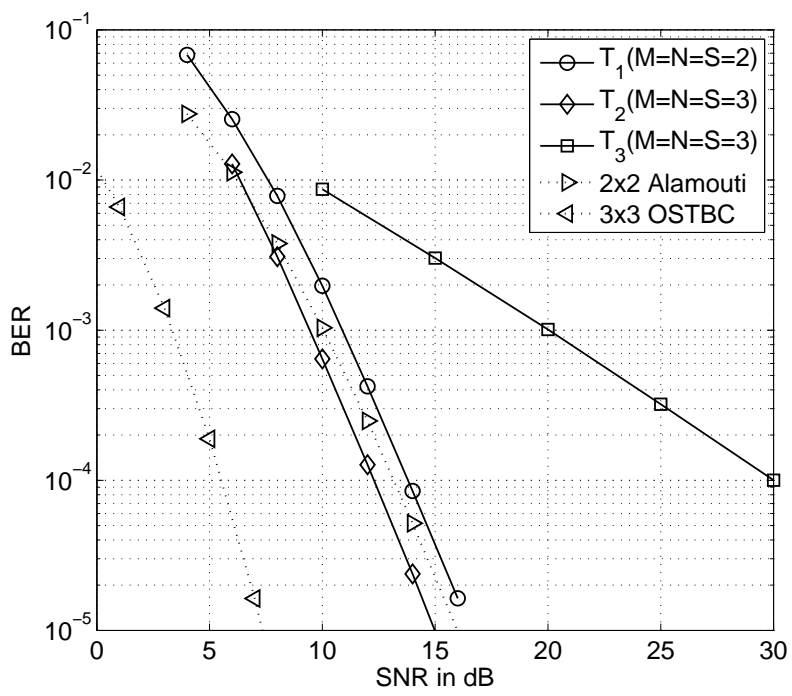

Fig. 3. Simulation Results for rate-1/2 convolutional code with different spatial de-multiplexer. 4-QAM is used for the reference curves.

mapping is used for the BICMB system. For the non-punctured code, the used generator polynomials are $(5,7)$ and $(5,7,7)$ for rates $1 / 2$ and $1 / 3$, respectively. For high rate codes such as $2 / 3$ and $3 / 4$, the perforation matrices in [9] are used from the $1 / 2$-rate original code. The well-known reference curves achieving the full diversity order of $M N$ are drawn from the Alamouti code for the $2 \times 2$ case and 1/2-rate orthogonal space-time block code (OSTBC) for the $3 \times 3$ case.

Fig. 3 shows BER performances for the cases corresponding to $\mathbf{T}_{1}, \mathbf{T}_{2}, \mathbf{T}_{3}$ in (10), (11), and (12). $Q_{\max }$ is found to be 1 for $\mathbf{T}_{1}$ because $\alpha_{1} \geq 1$ in all of the $\alpha$-vectors. In this case, as predicted by the analysis in [4], [5], the diversity order equals 4 by calculating (17) with $M=N=2$. From the figure, we can see that BER curve for $\mathbf{T}_{1}$ is parallel to that of $2 \times 2$ Alamouti code. Since $Q_{\max }$ for $\mathbf{T}_{2}$ is 2 due to the vector [0 053 ], the calculated diversity order is 4 in the case of $M=N=S=3$. This can be verified by Fig. 3. Although the same number of subchannels and same convolutional code are used, the spatial de-multiplexer, described in Section $\amalg$ for $\mathbf{T}_{\mathbf{3}}$, gives no diversity gain at all. The reason for this is that the vector $\left[\begin{array}{ll}0 & 0\end{array}\right]$ which can be observed from the transfer function in $\mathbf{T}_{3}$ makes $Q_{\max } 3$ resulting in the calculated diversity order of 1 in the equation (17) with $M=N=S=3$. This matches the simulation result.

Table \ shows the results of calculating the $\alpha$-vectors of BICMB system with a 4-state convolutional code and a simple rotating spatial de-multiplexer. $P$ and the dimension of the vector x according to the parameters $S$ and rate are shown. Then, the $\alpha$-vectors which have the largest $Q$ among the all $\alpha$-vectors are partly shown in the table. The value $Q_{\max }$ corresponding to the vectors found is also shown in the table.

Fig. 4 shows the BER performance of the punctured code with the same spatial de-multiplexer being used as that of the non-punctured code in the $2 \times 2$ case. Due to the puncturing 
TABLE I

Calculated Results of $\alpha$-Vectors

\begin{tabular}{|c|c|c|c|c|c|}
\hline$S$ & rate & $P$ & $\operatorname{dim}$ & vectors & $Q_{\max }$ \\
\hline \hline \multirow{3}{*}{2} & $1 / 2$ & 2 & 3 & & 1 \\
\cline { 2 - 6 } & $2 / 3$ & 6 & 12 & {$[05][08][09]$} & 2 \\
\cline { 2 - 6 } & $3 / 4$ & 4 & 9 & {$[04][05][06]$} & 2 \\
\hline \hline \multirow{4}{*}{3} & $1 / 3$ & 3 & 3 & & 1 \\
\cline { 2 - 6 } & $1 / 2$ & 6 & 9 & {$[053][046]$} & 2 \\
\cline { 2 - 6 } & $2 / 3$ & 3 & 6 & {$[012][022]$} & 2 \\
\cline { 2 - 6 } & $3 / 4$ & 12 & 27 & {$[005][008]$} & 3 \\
\hline
\end{tabular}

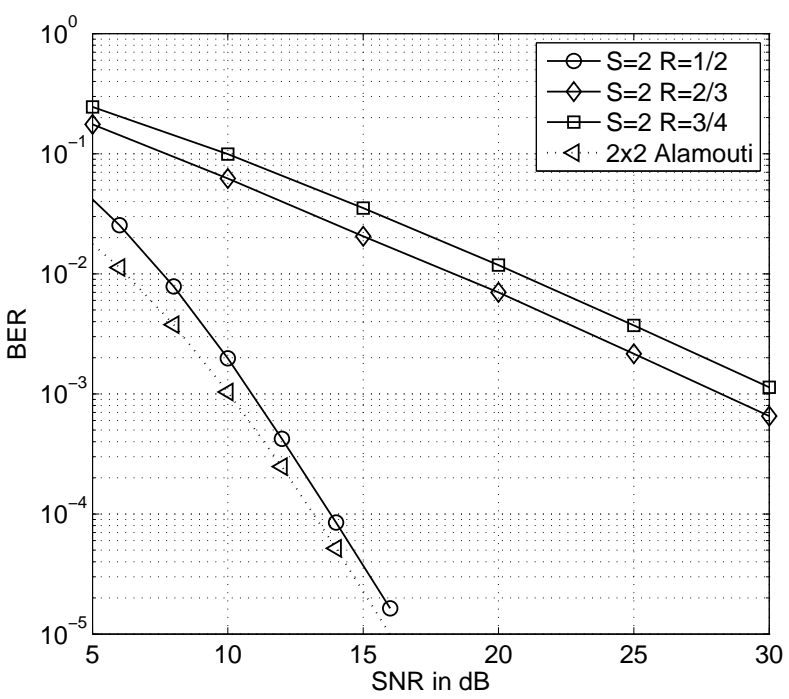

Fig. 4. Simulation Results for the $2 \times 2$ case where 4 -QAM is used for the Alamouti code

scheme, BICMB generates the $\alpha$-vectors whose $Q_{\max }$ equals 2 as shown in Table [ resulting in losing the the full diversity order.

As shown in Fig. 5, for a $3 \times 3$ system with 3 streams, only $1 / 3$-rate convolutional code achieves full diversity order of 9 since other codes have $Q_{\max }$ of larger than 1 as given in Table II The analytically calculated diversity orders by using (17) and Table \are 4, 4, 1 for $1 / 2,2 / 3,3 / 4$ respectively, which can be easily verified from Fig. 5 For the rate- $3 / 4$ code with the same spatial de-multiplexer, reducing one stream improves the performance dramatically. The diversity order of this case is 4 from the equation (17) with $M=N=3$ and $Q_{\max }=2$.

\section{CONCLUSION}

In this paper, we investigated the diversity order of BICMB when the interleaver does not achieve the previously introduced design criteria. We introduced a method to calculate the $\alpha$-vectors from a given convolutional code and a spatial de-multiplexer by using a transfer function. By using this method, the $\alpha$-vectors that do not fulfill the full diversity order criteria are quantified. Then, the diversity behavior corresponding to the $\alpha$-vectors was analyzed through PEP

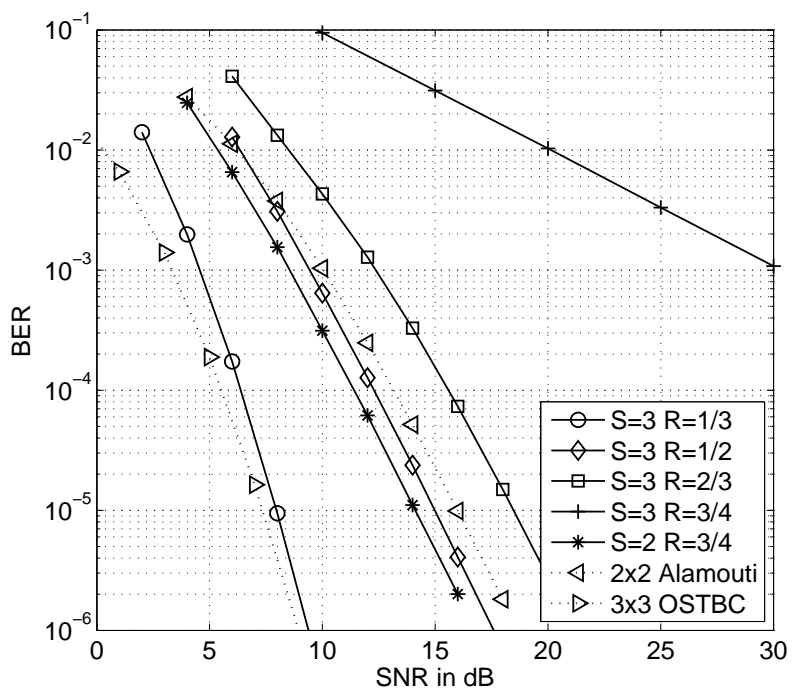

Fig. 5. Simulation Results for $3 \times 3 S=3$ case where 4 -QAM is used for both reference curves

calculation. The exponent of PEP between two codewords is $(M-Q+1)(N-Q+1)$ where $Q$ is the first index to the non-zero element in the $\alpha$-vector. Since BER is dominated by PEP with the smallest exponent, the diversity order is $\left(M-Q_{\max }+1\right)\left(N-Q_{\max }+1\right)$, where $Q_{\max }$ is the maximum among $Q$ 's corresponding to each $\alpha$-vector. We provided the simulation results that verify the analysis.

\section{REFERENCES}

[1] H. Jafarkhani, Space-Time Coding: Theory and Practice. Cambridge University Press, 2005.

[2] D. P. Palomar, J. M. Cioffi, and M. A. Lagunas, "Joint tx-rx beamforming design for multicarrier MIMO channels: A unified framework for convex optimization," IEEE Trans. Signal Process., vol. 51, no. 9, pp. 23812401, September 2003.

[3] E. Sengul, E. Akay, and E. Ayanoglu, "Diversity analysis of single and multiple beamforming," IEEE Trans. Commun., vol. 54, no. 6, pp. 990993, June 2006.

[4] E. Akay, E. Sengul, and E. Ayanoglu, "Bit interleaved coded multiple beamforming," IEEE Trans. Commun., vol. 55, no. 9, pp. 1802-1811, September 2007.

[5] E. Akay, H. J. Park, and E. Ayanoglu. On bit-interleaved coded multiple beamforming. ArXiv: 0807.2464. [Online]. Available: http://arxiv.org

[6] D. Haccoun and G. Begin, "High-rate punctured convolutional codes for viterbi and sequential decoding," IEEE Trans. Commun., vol. 37, no. 11, pp. 1113-1125, November 1989.

[7] E. Sengul, H. J. Park, and E. Ayanoglu, "Bit-interleaved coded multiple beamforming with imperfect CSIT," IEEE Trans. Commun., to appear.

[8] J. Conan, "The weight spectra of some short low-rate convolutional codes," IEEE Trans. Commun., vol. 32, no. 9, pp. 1050-1053, September 1984.

[9] G. Begin, D. Haccoun, and C. Paquin, "Further results on high-rate punctured convolutional codes for viterbi and sequential decoding," IEEE Trans. Commun., vol. 38, no. 11, pp. 1922-1928, November 1990.

[10] A. Khoshnevis and A. Sabharwal, "On diversity and multiplexing gain of multiple antenna systems with transmitter channel information," in Proc. Allerton Conference on Communication, Control and Computing, Monticello, IL, October 2004.

[11] K.-J. Lee and I. Lee, "Diversity analysis of coded SVD schemes for MIMO spatial multiplexing systems," in Proc. IEEE ICC '08, May 2008, pp. 4703-4707. 